\title{
二价双核铀聚吡咯配合物结构设计和铀-铀多重键的理论研究
}

\author{
陈方园 ${ }^{a}$ 曲宁 ${ }^{a}$ 吴群燕 ${ }^{b} \quad$ 张红星 $^{c}$ 石伟群*, $b$ 潘清江 $*, a$ \\ ( ${ }^{a}$ 黑龙江大学功能无机材料化学教育部重点实验室 化学化工与材料学院 哈尔滨 150080) \\ $\left({ }^{b}\right.$ 中国科学院高能物理研究所核能放射化学实验室 北京 100049) \\ ( ${ }^{c}$ 吉林大学理论化学研究所 长春 130023)
}

\begin{abstract}
摘要 经三十余年努力, 二价铀溶液化学于近年取得突破性进展, Evans 和 Meyer 等成功获得两个可通过 X-ray 晶体衍 射表征结构的有机金属铀(II)配合物. 为进一步拓展 $\mathrm{U}^{\mathrm{II}}$ 配合物化学和探索可能存在的金属多重键，设计双核配合物 $\left[\left(\mathrm{U}^{\mathrm{II}}\right)_{2}(\mathrm{~L})\right]$ ( $\mathrm{L}$ 为八齿氮供体低聚吡咯大环四价阴离子), 并使用相对论密度泛函理论优化其可能的电子自旋态异构体结 构和计算相关性质. 结果表明, $\left[\left(\mathrm{U}^{\mathrm{II}}\right)_{2}(\mathrm{~L})\right]$ 具有三重态基态, 其电子组态为 $\pi^{4} \sigma^{2} \delta^{2} ; \mathrm{U}(5 \mathrm{f})$ 原子轨道对高占据分子轨道有重 要贡献; 它的 $\mathrm{U}-\mathrm{U}$ 键长为 $2.33 \AA$ 、Mayer 键级为 3.89 和对应的伸缩振动频率为 $259 \mathrm{~cm}^{-1}$, 被指认为 $\mathrm{U}-\mathrm{U}$ 弱四重键. 这 一结论与 QTAIM (quantum theory of atoms in molecule) 的 $\mathrm{U}-\mathrm{U}$ 键临界点处的电子/能量密度拓扑分析结果相一致. 与不 同氧化态铀同类物 $\left[\left(\mathrm{U}^{m}\right)_{2}(\mathrm{~L})\right]^{n+}(m=\mathrm{III}, n=2 ; m=\mathrm{IV}, n=4)$ 比较显示, 随着铀氧化态增大, $\mathrm{U}-\mathrm{U}$ 距离增长、键级变小、 伸缩振动频率变小, 金属铀电子自旋密度与常规预期值的差值 $\left(\Delta S_{\mathrm{U}}\right)$ 增大; 结合分子轨道和 QTAIM 参数分析, 发现金属 氧化态可以调控配体和金属轨道能级匹配程度和改变金属-金属多重键.
\end{abstract}

关键词 双核 UII 聚吡咯配合物; 电子结构; 铀-铀多重键; QTAIM 拓扑分析; 相对论密度泛函理论

\section{Structures and Uranium-Uranium Multiple Bond of Binuclear Divalent Uranium Complex of Pyrrolic Schiff-base Macrocycle: a Relativistic DFT Probe}

\author{
Chen, Fangyuan ${ }^{a}$ \\ Qu, Ning ${ }^{a}$ \\ Wu, Qunyan ${ }^{b}$ \\ Pan, Qingjiang*,a
}

( ${ }^{a}$ Key Laboratory of Functional Inorganic Material Chemistry of Education Ministry, School of Chemistry and Materials Science, Heilongjiang University, Harbin 150080)

$\left({ }^{b}\right.$ Laboratory of Nuclear Energy Chemistry, Institute of High Energy Physics, Chinese Academy of Sciences, Beijing 100049, China)

( ${ }^{c}$ Institute of Theoretical Chemistry, Jilin University, Changchun 130023)

\begin{abstract}
Although attempts to synthesize divalent uranium molecules were begun three decades ago, molecular U(II) species isolable in solution have been not achieved until recent years. In 2013, Evans and co-workers synthesized the first U(II) complex, $\left[\mathrm{U}\left(\mathrm{Cp}^{\prime}\right)_{3}\right] \cdot[\mathrm{K}(2,2,2$-cryptand $)]\left(\mathrm{Cp}^{\prime}=\mathrm{C}_{5} \mathrm{H}_{4} \mathrm{SiMe}_{3}\right)$ via flash reduction, that was suitable for X-ray crystal diffraction characterization. A year later, the group of Meyer obtained another divalent uranium complex, $\left[\mathrm{U}\left(\left({ }^{\mathrm{Ad}, \mathrm{Me}} \mathrm{ArO}\right)_{3} \mathrm{mes}\right)\right] \cdot[\mathrm{K}(2,2,2$-cryptand $)]$ employing their particularly interesting tris(aryloxide) arene ligand. The $5 \mathrm{f}^{3} 6 \mathrm{~d}^{1}$ and $5 \mathrm{f}^{4}$ ground states were assigned to these two complexes, respectively, by the jointed experimental/theoretical studies. It was demonstrated that the ligand significantly affect the nature of the ground state of divalent uranium complex by tuning the energetic separation of the $5 \mathrm{f}$ and $6 \mathrm{~d}$ orbitals. Therefore, careful selection of ligand makes it possible to have access to $+\mathrm{II}$ oxidation state of uranium and prepare new $U^{I I}$ complex. A flexible octadentate polypyrrollic Schiff-base macrocycle $\left(\mathrm{H}_{4} \mathrm{~L}\right)$ has been developed to complex a variety of metals such as actinides, rare earth and transition metals that show a wide range of size and diverse oxidation states. Both mono- and bimetallic complexes featured with an intriguing "Pacman-like" structure were obtained. For example, the reaction of $\mathrm{H}_{4} \mathrm{~L}$ with a trivalent uranium precursor $\left[\left(\mathrm{U}^{\mathrm{III}}\right) \mathrm{I}_{3}(\mathrm{THF})_{4}\right]$ yielded a neutral $\left[\left(\mathrm{U}^{\mathrm{IV}}\right)(\mathrm{L})\right]$ complex, where the uranium ion was determined by the single crystal X-ray diffraction to be situated inside the ligand mouth and held by eight nitrogen atoms together. The +IV oxidation state was assigned to the uranium by presuming dihydrogen elimination. Considering the flexibility, tetravalent-anion nature as well as capability of accommodating bimetallic ions and stabilizing various oxidation states of uranium (e.g. III $\sim$ VI complexes have been found so far) that the polypyrrolic ligand has exhibited in previously synthesized complexes, two divalent uranium ions would be likely complexated by the ligand to generate a complex, $\left[\left(\mathrm{U}^{\mathrm{II}}\right)_{2}(\mathrm{~L})\right]$. In addition to enriching the coordination chemistry of $\mathrm{U}(\mathrm{II})$, it is also a good example to explore electronic structures of the low-valent uranium complex and unravel the uranium-uranium multiple
\end{abstract}

*E-mail: panqjitc@163.com (QJ Pan),shiwq@ihep.ac.cn (WQ Shi)

Received January 9, 2017; published March 27, 2017.

Supporting information for this article is available free of charge via the Internet at http://sioc-journal.cn.

Project supported by the National Natural Science Foundation of China (Nos. 21273063, 21477130) and the Scientific Foundation of Heilongjiang Province for the Returned Overseas Chinese Scholars.

项目由国家自然科学基金(Nos. 21273063, 21477130)和黑龙江省留学回国人员科技项目择优资助. 
bonding nature. Although many theoretical studies have explored uranium complexes, the study focusing on the divalent diuranium complex of a single macrocyclic ligand remains rare. In the work, a relativistic density functional theory has been employed to investigate $\left[\left(\mathrm{U}^{\mathrm{II}}\right)_{2}(\mathrm{~L})\right]$. The structures in electron spin states (singlet, triplet, quintet, septet and nonet) were optimized. Short distances of $U-U(2.32 \sim 2.67 \AA)$, large bond order $(2.95 \sim 3.90)$ and high stretching vibrational frequencies $\left(180 \sim 263 \mathrm{~cm}^{-1}\right)$ were calculated. Energetic calculations find that its triplet state is the ground state. It has the electronic configuration of $\pi^{4} \sigma^{2} \delta^{2}$, primarily contributed by $\mathrm{U}(5 \mathrm{f})$ character. Structural and molecular-orbital analyses suggest a slightly weak uranium-uranium quadruple bond, which is confirmed by the quantum theory of atoms in molecule (QTAIM) calculations. Further comparison with analogues $\left[\left(\mathrm{U}^{\mathrm{III}}\right)_{2}(\mathrm{~L})\right]^{2+}$ and $\left[\left(\mathrm{U}^{\mathrm{IV}}\right)_{2}(\mathrm{~L})\right]^{4+}$ was also addressed. It is found that the uranium oxidation state is able to tune the energetic matching between the highest-energy occupied orbital of ligand and the adjacent low-energy metal-based orbital, as well as correlates with the electron transfer between metal and ligand and the diuranium multiple bond number.

Keywords bimetallic $\mathrm{U}^{\mathrm{II}}$ polypyrrolic complexes; electronic structures; diuranium multiple bond; QTAIM; relativistic DFT

\section{1 引言}

铀是核能生产、核武器制造和核废料处理的最重要 元素之一 ${ }^{[1]}$. 目前已知铀的氧化态包括 II $\sim$ VI. 相比其 它氧化态，在溶液化学中稳定存在的二价铀分子配合物 少之又少 ${ }^{[2 \sim 4]}$. 虽然从 20 世纪 80 年代就开始二价铀化 合物溶液化学(配位化学和有机金属化学)研究, 但是直 到四年前才合成第一个已知结构的二价铀配合物. 即, Evans 课题组 2013 年采用低温还原有机金属铀化合物 $\left[\mathrm{U}^{\mathrm{III}}\left(\mathrm{Cp}^{\prime}\right)_{3}\right]\left(\mathrm{Cp}^{\prime}=\mathrm{C}_{5} \mathrm{H}_{4} \mathrm{SiMe}_{3}\right)$ 方法成功制备可适用于 X-ray 晶体衍射技术表征的二价铀金属配合物 $\left[\mathrm{U}^{\mathrm{II}}\left(\mathrm{Cp}^{\prime}\right)_{3}\right] \cdot[\mathrm{K}(2,2,2 \text {-cryptand })]^{[3]}$; 文中的理论计算和实 验表征共同揭示该配合物具有 $5 \mathrm{f}^{3} 6 \mathrm{~d}^{1}$ 的基态电子组态, 与以前发现的二价稀土配合物 $4 \mathrm{f}^{\mathrm{n}} 5 \mathrm{~d}^{1}$ 基态电子结构类 似, 但不同于二价铀离子 $5 \mathrm{f}^{4}$ 结构 ${ }^{[5]}$. 一年后, 德国 Meyer 教授及其合作者使用类似合成方法、采用他们独 特的 tris(aryloxide) arene 类配体 $\left({ }^{\mathrm{Ad}, \mathrm{Me}} \mathrm{ArO}\right)_{3}$ mes 得到配合 物 $\left[\mathrm{U}^{\mathrm{II}}\left(\left(^{\mathrm{Ad}, \mathrm{Me}} \mathrm{ArO}\right)_{3} \mathrm{mes}\right)\right] \cdot[\mathrm{K}(2,2,2 \text {-cryptand })]^{[4]}$; 有趣的是, 这个化合物基态电子组态为 $5 \mathrm{f}^{4}$. 以上研究表明, 配体能 够调控 $5 \mathrm{f}$ 和 $6 \mathrm{~d}$ 轨道能级, 影响二价铀配合物基态电子 结构, 同时它也是稳定二价铀离子的重要因素之一 ${ }^{[2]}$. 因此, 选择适当配体有望能够合成更多的新型二价铀配 合物.

柔性八齿氮配位低聚吡咯配体大环 $\left(\mathrm{H}_{4} \mathrm{~L}\right.$, 图 1)分别 由 Sessler 教授 ${ }^{[6]}$ 和 Love 教授 ${ }^{[7]}$ 课题组合成. 后者进一步 使用该配体成功络合种类多样和氧化态各异的金属离 子, 包括钶系、稀土和过渡金属, 获得具有 “Pacman” 结构的单核和双核金属配合物 ${ }^{[8 \sim 13]}$. 值得指出的是, 这 些配合物中涵盖从 III IV 的金属铀. 就配位结构而言, 大环配体既可使用它的两个 $\mathrm{N}_{4}$ 供体空穴同时配位两个 金属离子, 也可只用一个 $\mathrm{N}_{4}$ 空穴配位一个金属而另一 个空穴空置, 还可以直接由八个 $\mathrm{N}$ 包裹一个金属离子. 例如，通过 $\mathrm{H}_{4} \mathrm{~L}$ 和 $\left[\left(\mathrm{U}^{\mathrm{III}}\right) \mathrm{I}_{3}(\mathrm{THF})_{4}\right]$ 反应合成 $\left[\left(\mathrm{U}^{\mathrm{IV}}\right)(\mathrm{L})\right]$ 配 合物; X-ray 晶体衍射表明 $\mathrm{U}^{\mathrm{IV}}$ 同时被八个 $\mathrm{N}$ 原子配位 [10]. 既然 $\mathrm{H}_{4} \mathrm{~L}$ 低聚吡咯大环在配合物中形成负四价阴离 子、具有柔软结构特性且可以同时络合两个金属离子和 稳定各种金属氧化态(已知有 $\mathrm{U}^{\mathrm{III}} \sim \mathrm{U}^{\mathrm{VI}}$ 配合物), 那么可 否使用该配体同时络合两个二价铀金属离子呢? 配合
物 $\left[\left(\mathrm{U}^{\mathrm{II}}\right)_{2}(\mathrm{~L})\right]$ 的研究不仅可以丰富二价铀配位化学和其 几何/电子结构, 也为探索铀-铀金属多重键提供很好的 模型化合物.
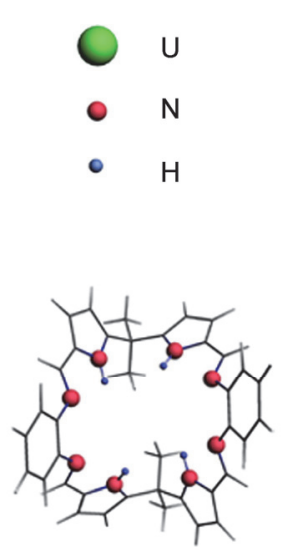

$\mathrm{H}_{4} \mathrm{~L}$
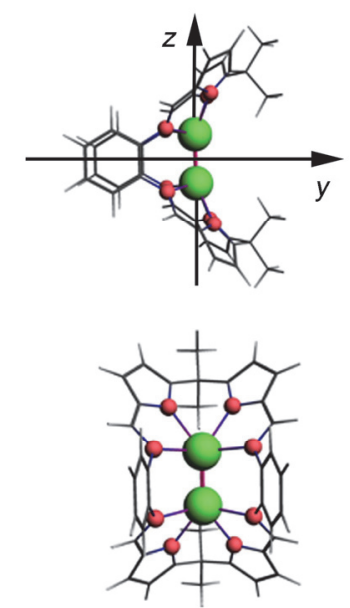

$\left[\left(\mathbf{U}^{m}\right)_{2}(L)\right]^{n+}$
图 1 用 Priroda 程序在 PBE/B-I 方法下优化的 $\mathrm{H}_{4} \mathrm{~L}$ 配体和 $\left[\left(\mathrm{U}^{m}\right)_{2}(\mathrm{~L})\right]^{n^{+}}$ $(m=\mathrm{II} \sim \mathrm{IV}, n=0 \sim 4)$ 配合物结构, 其中分别给出了配合物的侧视图 (上)和正视图(下)

Figure 1 Optimized structures (left) of the ligand $\mathrm{H}_{4} \mathrm{~L}$ and the complexes $\left[\left(\mathrm{U}^{m}\right)_{2}(\mathrm{~L})\right]^{n+}(m=\mathrm{II} \sim \mathrm{IV}, n=0 \sim 4)$ in Priroda codes where side-on (top) and face-on (bottom) views are shown

因其在乏燃料、核废料等中的存在形式，且极易溶 于水对环境造成很大的污染，各种氧化态铀化合物溶液 化学研究非常重要. 本文设计的双核铀聚吡咯配合物, 通过对其稳定性和化学键性质探索, 将对铀配合物的分 离化学、降低核废料对环境的污染等方面的实验开展具 有理论指导意义.

\section{2 计算方法}

理论设计和计算双核金属铀配合物 $\left[\left(\mathrm{U}^{m}\right)_{2}(\mathrm{~L})\right]^{n+}$ ( $m=\mathrm{II}, n=0 ; m=\mathrm{III}, n=2 ; m=\mathrm{IV}, n=4)$. 最近, 潘清江 课题组 ${ }^{[14]}$ 报道了三价配合物多样化的空间异构体结构, 鉴于本文目的是探索低价化合物电子性质(尤其是二价 化合物)和铀-铀金属多重键, 所以我们将关注两个铀原 子通过 $z$ 轴的空间异构体, 如图 1 所示. 由于低氧化态 
铀含多个 $5 \mathrm{f}$ 和/或 $6 \mathrm{~d}$ 电子, 其双核配合物可形成多种电 子自旋态. 例如, $\left[\left(\mathrm{U}^{\mathrm{II}}\right)_{2}(\mathrm{~L})\right]$ 包括从单重态到九重态共计 五种可能电子自旋态. 本文将优化二价和四价配合物所 有电子自旋态结构, 并与三价化合物的进行比较讨论.

采用 Priroda 程序优化配合物在各种自旋态下的“异 构体” 结构. 该程序运用全电子四分量标量相对论(AE) 哈密顿 ${ }^{[15]}$, 采用广义梯度近似的 PBE (Perdew-BurkeErnzerh)泛函 ${ }^{[16]}$ 和全电子 correlation-consistent Gaussian 基组(用 B-I 表示). 在优化结构基础上进行频率计算, 未 得到任何虚频表明优化的结构是稳定构型.

进而, 使用 ADF2014 程序包 ${ }^{[17]}$ 探索双核铀配合物 基态电子性质. 为描述配合物的分子环境, 计算中采用 COSMO (Conductor-like Screening Model) ${ }^{[18]}$ 模型, 选用 $\mathrm{THF}$ 溶剂, 介电常数为 7.58; 模型中 Klamt 半径为 $\mathrm{U}=$ $1.70 \AA 、 \mathrm{~N}=1.83 \AA 、 \mathrm{C}=2.00 \AA$ 和 $\mathrm{H}=1.30 \AA^{[14,18 \sim 20]}$. ADF 计算中使用标量相对论 ZORA (Zero Order Regular Approximation)哈密顿 ${ }^{[21]}$ 、Slater 型 TZP 基组(B-II)和 PBE 泛函, 并对 $U$ 和 $N / C$ 分别采用冻结 $1 \mathrm{~s} \sim 4 \mathrm{f}$ 和 $1 \mathrm{~s}$ 核轨道 近似. 分别使用 ADF 和 Priroda 程序计算 Mayer 键级和 Mulliken 电荷, 前者计算还得到原子轨道布居. 为进一 步考察泛函选择对这些计算结果的影响, 还使用 ADF 程序中的杂化泛函 B3LYP(包含 $20 \%$ HF exchange)和 mPW1PW(42.8\%)进行计算. 最后, 采用 ADF 程序进行 Quantum Theory of Atoms in Molecule (QTAIM)计算, 得 到 $\mathrm{U}-\mathrm{U}$ 键临界点(Bond critical point)的电子密度 $\rho(r)$ 和 拉普拉斯密度 $\nabla^{2} \rho(r)$; 同时采用 Gaussian09 程序 ${ }^{[22]}$, 运 用 6-31G (d)基组、B3LYP 泛函方法、其中 U 原子使用 准相对论效应的小核噟势 ECP60MWB ${ }^{[23]}$, 结合 Multiwfn 软件分析得到能量密度 $H(r)^{[24]}$.

\section{3 结果与讨论}

\section{1 几何结构和相对稳定性}

在表 1 中, 列出了优化得到配合物 $\left[\left(\mathrm{U}^{m}\right)_{2}(\mathrm{~L})\right]^{n+}(m$ $=\mathrm{II} \sim \mathrm{IV}, n=0 \sim 4)$ 所有电子自旋态的 $\mathrm{U}-\mathrm{U}$ 键长、键级、 伸缩振动频率等信息, 并于图 1 中给出其结构图. 可以 看出, 二价铀配合物的 U-U键长从单重态的 $2.32 \AA$ 增长 至九重态的 $2.67 \AA$, 对应的 Mayer 键级则从 3.90 逐渐减 少至 2.95; 同时, 频率计算发现 $U-\mathrm{U}$ 伸缩振动在 180 $263 \mathrm{~cm}^{-1}$ 范围. 通过这些数据可以推断出二价 $\mathrm{U}-\mathrm{U}$ 之 间具有金属-金属多重键, 其强度介于三重键和四重键 之间. 其对应的优化四价铀配合物的单重态、三重态和 五重态结构中的 $U-U$ 键长分别为 $2.63 、 2.64$ 和 $2.72 \AA$, 其对应的键级 $(2.28 \sim 2.33)$ 和 $\mathrm{U}-\mathrm{U}$ 伸缩振动频率 $(140 \sim$ $156 \mathrm{~cm}^{-1}$ ); 两个 $\mathrm{U}^{\mathrm{IV}}$ 金属键可能具有双重键性质. 很明 显, 三价铀配合物的各自旋态结构中的 $U-U$ 键长 (2.43 2.62 A)、键级(3.08 3.18)和伸缩振动频率(188 $220 \mathrm{~cm}^{-1}$ ) 表明其 $U-U$ 键具有三重键特征. 到目前为止, 虽然实验已合成一些含 $U-M(M$ 为过渡金属和主族金

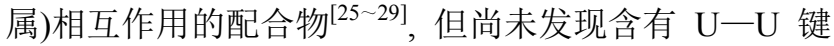
的配合物. 另外, 理论计算对双核 $U^{\mathrm{III}}$ 配合物(分子)的研 究较多, 如 $\mathrm{U}^{\mathrm{III}}{ }_{2} \mathrm{X}_{6}$ 和 $\left[\mathrm{U}^{\mathrm{III}}{ }_{2} \mathrm{X}_{8}\right]^{2-}\left(\mathrm{X}=\mathrm{F}, \mathrm{Cl}, \mathrm{OH}, \mathrm{NH}_{2}\right.$ 和

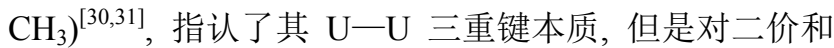
四价双核铀配合物的报道却很少 ${ }^{[32,33]}$.

表 1 计算配合物在不同电子自旋态 $(\mathrm{ESS})$ 下 $\mathrm{U}-\mathrm{U}$ 的键长 $(D, \AA)$ 、键 级 $(\mathrm{BO})$ 、伸缩振动频率 $\left(v, \mathrm{~cm}^{-1}\right)$ 和铀原子电子自旋密度 $\left(S_{\mathrm{U}}\right)$, 以及它们 的相对能量 $(\mathrm{kJ} / \mathrm{mol})^{a}$

Table 1 Calculated $\mathrm{U}-\mathrm{U}$ distances $(D, \AA)$, bond orders (BO), stretching vibrational frequencies $\left(v, \mathrm{~cm}^{-1}\right)$ and electron-spin density $\left(S_{\mathrm{U}}\right)$ of uranium atoms for diuranium complexes in various electron-spin states (ESS), together with the relative energies $(\mathrm{kJ} / \mathrm{mol})^{a}$

\begin{tabular}{ccccccccc}
\hline Complex & ESS & $D$ & $v$ & BO & $S_{\mathrm{U}}$ & $Q_{\mathrm{U}}$ & $\Delta E$ & $\Delta E_{0}$ \\
\hline$\left[\left(\mathrm{U}^{\mathrm{II}}\right)_{2}(\mathrm{~L})\right]$ & nonet & 2.668 & 180 & 2.95 & 3.789 & 1.055 & 68.0 & 64.9 \\
& septet & 2.517 & 196 & 3.52 & 2.958 & 1.078 & 42.5 & 40.1 \\
& quintet & 2.412 & 235 & 3.76 & 1.916 & 1.087 & 27.7 & 25.8 \\
& triplet & 2.332 & 259 & 3.89 & 0.939 & 1.067 & 0.0 & 0.0 \\
& singlet & 2.324 & 263 & 3.90 & 0.000 & 1.069 & 24.4 & 23.9 \\
{$\left[\left(\mathrm{U}^{\mathrm{III}}\right)_{2}(\mathrm{~L})\right]^{2+}$} & septet & 2.622 & 188 & 3.08 & 3.001 & 1.372 & 26.9 & 26.9 \\
& quintet & 2.506 & 201 & 3.14 & 2.045 & 1.373 & 2.8 & 2.3 \\
& triplet & 2.474 & 214 & 3.18 & 1.073 & 1.380 & 0.0 & 0.0 \\
& singlet & 2.426 & 220 & 3.17 & 0.000 & 1.349 & 11.9 & 12.3 \\
{$\left[\left(\mathrm{U}^{\mathrm{IV}}\right)_{2}(\mathrm{~L})\right]^{4+}$} & quintet & 2.720 & 140 & 2.28 & 2.103 & 1.541 & 0.0 & 0.0 \\
& triplet & 2.641 & 149 & 2.33 & 1.240 & 1.516 & 11.8 & 11.8 \\
& singlet & 2.627 & 156 & 2.31 & 0.000 & 1.528 & 31.8 & 31.8 \\
\hline
\end{tabular}

${ }^{a} \Delta E$ denotes the total energy and $\Delta E_{0}$ includes the zero-point vibration energy, which were obtained by subtracting the respective ground-state energy. Trivalent complexes were reported in our previous work ${ }^{[14]}$.

表 1 和图 1 给出了各种电子自旋态异构体的相对能 量. 可以看出, 二价铀配合物的三重态能量最低, 为基 态. 它比对应的其它态的总能量 $(\Delta E)$ 低 $24 \sim 68 \mathrm{~kJ} / \mathrm{mol}$; 包括零点振动能的能量 $\left(\Delta E_{0}\right)$ 表现出同样的趋势. 四价 铀配合物的五重态为基态, 比其单重态和三重态分别低 32 和 $12 \mathrm{~kJ} / \mathrm{mol}(\Delta E)$. 能量计算表明三价铀配合物的三 重态的能量最低, 但是仅仅比其五重态低 $3 \mathrm{~kJ} / \mathrm{mol}$, 说 明这两个电子自旋态很容易在室温下相互转换. 以二价 铀配合物为例, 使用不同泛函(Pri: B3LYP)和不同程序 (ADF: PBE 和 B3LYP)计算其各个自旋态的相对能量(见 支持信息表 $\mathrm{S} 1$ ), 得到结果均表明三重态为二价配合物 的基态. 最近 Truhlar 教授课题组 ${ }^{[34]}$ 发展的 Minnesota 系 列泛函能够在较低计算资源消耗时得到与高级别波函 数理论方法 [如 $\operatorname{CCSD}(\mathrm{T})]$ 一样准确的体系能量, 有望在 将来用于双核铀体系的计算.

考虑到理论方法对化学键强度的影响, 我们使用 $\mathrm{ADF}$ 程序计算上述三种配合物基态的 $\mathrm{U}-\mathrm{U}$ 和 $\mathrm{U}-\mathrm{N}$ 键 级(表 2). 不难看出, ADF 计算的二价铀配合物 $U-U$ 键 的 Mayer 键级为 4.80, 远大于 Priroda 得到的 3.89; 而前 者得到 $\mathrm{U}-\mathrm{N}$ 平均键级为 0.39 , 小于后者 $(0.51)$. 可以推 断出, 由于两种理论级别采用不同基组( 分别用 Gaussian 和 Slater 基组)和相对论哈密顿(AE 和 ZORA), 所以即使都运用同样的泛函(PBE)和键级 Partition 方法 (Mayer)也导致键级数据的差别. 即在 ADF 计算中趋于 
高估电子密度在 $\mathrm{U}-\mathrm{U}$ 间的分布, 而使得 $\mathrm{U}-\mathrm{N}$ 键级相 对较小; 而 Priroda 则是倾向于电子在 $\mathrm{U}-\mathrm{N}$ 间的分布, 使得 $U-U$ 键级相对较小. 同样的, ADF 和 Priroda 计算 的三价铀配合物的 $\mathrm{U}-\mathrm{U}$ 和 $\mathrm{U}-\mathrm{N}$ 键级也有很大差别, 显然还是电子密度分布所致. 有趣的是, 四价铀配合物 的 $U-U$ 键级在两种理论级别下的计算结果非常接近. 通过三个配合物比较来看, 在二价和三价铀配合物时, 金属富电子导致 $\mathrm{U}-\mathrm{U}$ 周围电子密度与 $\mathrm{U}-\mathrm{N}$ 周围差异 较大, 所以计算条件(如基组、哈密顿)变化对键级结果 影响显著; 而四价铀配合物中, $\mathrm{U}-\mathrm{U}$ 和 $\mathrm{U}-\mathrm{N}$ 周边电子 密度差异不再像低价化合物那么明显, 所以 ADF 和 Priroda 的计算结果也相对接近. 另外, 从表 2 可以看到, ADF 计算的 Gophinatan-Jug (G-J) 和不同级别 Nalewajski-Mrozek [N-M(i)] 与其计算的 Mayer 键级也有 差异. 为进一步考察泛函的影响, 使用杂化泛函 B3LYP 和 mPW1PW(ADF 程序)计算的二价铀配合物 $\mathrm{U}-\mathrm{U}$ 的 Mayer 键级分别为 3.65 和 3.67, 比 PBE 计算值低很多, 但是其平均 $\mathrm{U}-\mathrm{N}$ 键级值则相对较高. 值得指出的是, 这一数值却接近 Priroda 的计算结果. 总之, 键级的计算 结果与使用的基组(Gaussian 和 Slater)、相对论哈密顿 (AE 和 ZORA)、密度泛函方法(PBE、B3LYP 和 mPW1PW) 和键级密度 Partition 处理方法(Mayer、G-J 和 N-M)有关.

\section{2 原子电荷、电子自旋密度和轨道布居}

Priroda 程序计算的 $\left[\left(\mathrm{U}^{m}\right)_{2}(\mathrm{~L})\right]^{n+} \quad(m=\mathrm{II} \sim \mathrm{IV}, n=0 \sim$ 4) 的铀原子电荷 $\left(Q_{\mathrm{U}}\right)$ 分别为 $1.06 \sim 1.09 、 1.35 \sim 1.38$ 和 $1.52 \sim 1.53$ (表 1). 很明显, 这些数值与金属正常氧化态 偏离很多, 但随着铀原子氧化态增加而增大. 相比之下, 得到的铀原子电子自旋密度 $\left(S_{\mathrm{U}}\right)$ 与其预期常规数值偏差 较小. 图 2 绘制出这一差值 $\left(\Delta S_{\mathrm{U}}\right)$ 与电子自旋态的相关 图. 可以看出, 二价铀配合物所有电子自旋态的 $\Delta S_{\mathrm{U}}$ 均 为负值, 表明电子密度由金属流向配体; 而其他两类铀 配合物的 $\Delta S_{\mathrm{U}}$ 均为正值, 显示有更多电子密度定域到金 属上, 且四价的电子转移大于三价; 因此, 整体呈现 $\Delta S_{\mathrm{U}}$ 数值随着金属氧化态增大而增大的规律性变化. 大
部分铀配合物电子自旋态的 $\Delta S_{\mathrm{U}}$ 小于 0.1 , 表明其 $S_{\mathrm{U}}$ 能 够反映其电子自旋态结构; 二价铀配合物九重态和四价 铀配合物的三重态的 $S_{\mathrm{U}}$ 分别为 3.79 和 1.24 , 与常规预 期值相差最大, 对应的差值 $\Delta S_{\mathrm{U}}$ 分别为 0.21 和 0.24 .

表 3 列出了 $\mathrm{ADF}$ 对配合物基态铀的电荷 $\left(Q_{\mathrm{U}}\right)$ 和电 子自旋密度 $\left(S_{\mathrm{U}}\right)$ 的计算结果. 就铀的电荷而言, ADF 所 得数值略高于 Priroda 计算结果, 由于铀电荷计算受基 组影响很大, 所以它们之间的不同可归结为基组差异引 起. ADF 计算二价、三价和四价铀配合物基态的 $S_{\mathrm{U}}$ 分别 为 $0.96 、 1.11 、 2.13$, 与 Priroda 计算相差不到 0.04. 对 二价铀配合物的 $S_{\mathrm{U}}$ 数值而言, 使用 $\mathrm{ADF}$ 的杂化泛函 (B3LYP 和 $\mathrm{mPW} 1 \mathrm{PW}$ )方法得到的数值与 PBE 方法的非 常接近, 偏差不到 0.02 . 可以看出, 电子自旋密度不但 能够比电荷更好的描述配合物的电子自旋态, 而且不受 基组和密度泛函的影响.

使用 ADF 方法分析了不同铀氧化态配合物基态的 金属原子布居. $\mathrm{PBE}$ 方法计算的 $\mathrm{U}^{\mathrm{II}}$ 的电子组态为 $5 \mathrm{f}^{3.15} 6 \mathrm{~s}^{1.90} 6 \mathrm{p}^{6.00} 6 \mathrm{~d}^{1.82} 7 \mathrm{p}^{0.06}$; 随着铀氧化态的增加, $\mathrm{U}^{\mathrm{III}}$ 的 $5 f$ 和 $6 p$ 轨道分别减少 0.11 和 0.16 个电子，而 $6 s$ 和 $6 \mathrm{~d}$ 轨道中电子变化不大; 从三价铀到四价铀, $6 \mathrm{~s}$ 轨道电子

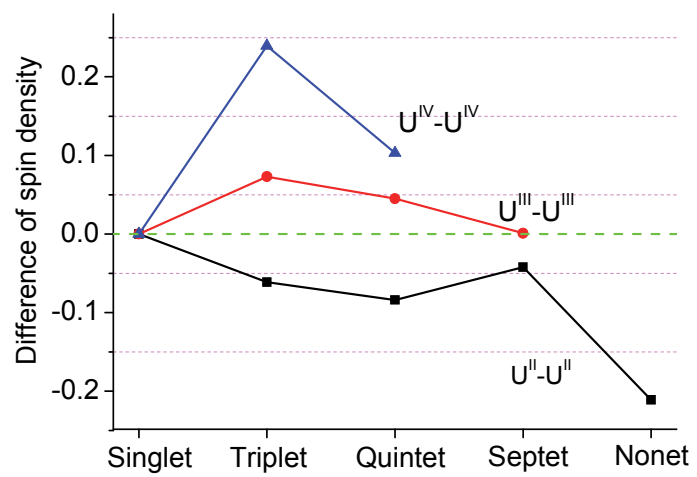

图 2 计算的 $\left[\left(\mathrm{U}^{m}\right)_{2}(\mathrm{~L})\right]^{n^{+}}(m=\mathrm{II}, n=0 ; m=\mathrm{III}, n=2 ; m=\mathrm{IV}, n=4)$ 不 同电子自旋异构体中铀自旋密度值与其期望值的差值, 即单重态、三 重态、五重态、七重态和九重态的期望值分别为 $0 、 1 、 2 、 3$ 和 4 Figure 2 Difference $\left(\Delta S_{\mathrm{U}}\right)$ of spin density of uranium atom of $\left[\left(\mathrm{U}^{m}\right)_{2}(\mathrm{~L})\right]^{n+} \quad(m=\mathrm{II}, n=0 ; m=\mathrm{III}, n=2 ; m=\mathrm{IV}, n=4)$ in various electronic spin states from the respective expected formal value, i.e. $0,1,2,3$ and 4 for the singlet, triplet, quintet, septet and nonet states, respectively.

表 2 各种泛函计算的双金属铀配合物基态 $\mathrm{U}-\mathrm{U}$ 和 $\mathrm{U}-\mathrm{N}$ 键长 $(D, \AA)$ 和键级

Table 2 Calculated $\mathrm{U}-\mathrm{U}$ and $\mathrm{U}-\mathrm{N}$ bond distances $(D, \AA)$ and bond orders of the diuranium complexes in their ground states

\begin{tabular}{|c|c|c|c|c|c|c|c|c|c|}
\hline \multirow{2}{*}{ Complex } & \multirow{2}{*}{ Functional } & \multirow{2}{*}{ Bond } & \multirow{2}{*}{$D$} & \multicolumn{5}{|c|}{ ADF: DFT } & \multirow{2}{*}{$\begin{array}{c}\text { Pri: DFT } \\
\text { Mayer }\end{array}$} \\
\hline & & & & Mayer & G-J ${ }^{a}$ & $\mathrm{~N}-\mathrm{M}(1)^{b}$ & $\mathrm{~N}-\mathrm{M}(2)^{b}$ & $\mathrm{~N}-\mathrm{M}(3)^{b}$ & \\
\hline \multirow[t]{6}{*}[(\mathrm{U}^{\mathrm{II}})_{2}(\mathrm{L})]{} & PBE & $\mathrm{U}-\mathrm{U}$ & 2.332 & 4.80 & 4.13 & 4.94 & 4.54 & 4.67 & 3.89 \\
\hline & & $(\mathrm{U}-\mathrm{N})_{\mathrm{av} .}$ & 2.433 & 0.39 & 0.62 & 0.70 & 0.81 & 0.70 & 0.51 \\
\hline & B3LYP & $\mathrm{U}-\mathrm{U}$ & 2.332 & 3.65 & 4.15 & 5.11 & 4.46 & 4.75 & \\
\hline & & $(\mathrm{U}-\mathrm{N})_{\mathrm{av} .}$ & 2.433 & 0.45 & 0.57 & 0.66 & 0.82 & 0.66 & \\
\hline & mPW1PW & $\mathrm{U}-\mathrm{U}$ & 2.332 & 3.67 & 4.17 & 5.17 & 4.52 & 4.80 & \\
\hline & & $(\mathrm{U}-\mathrm{N})_{\mathrm{av} .}$ & 2.433 & 0.43 & 0.57 & 0.66 & 0.81 & 0.66 & \\
\hline \multirow[t]{2}{*}[(\mathrm{U}^{\mathrm{III}})_{2}(\mathrm{L})]{$^{2+}$} & PBE & $\mathrm{U}-\mathrm{U}$ & 2.474 & 3.56 & 3.40 & 4.15 & 3.37 & 3.81 & 3.18 \\
\hline & & $(\mathrm{U}-\mathrm{N})_{\mathrm{av} .}$ & 2.352 & 0.46 & 0.74 & 0.85 & 0.99 & 0.84 & 0.63 \\
\hline \multirow[t]{2}{*}[(\mathrm{U}^{\mathrm{IV}})_{2}(\mathrm{L})]{$^{4+}$} & PBE & $\mathrm{U}-\mathrm{U}$ & 2.720 & 2.32 & 2.45 & 2.81 & 1.86 & 2.53 & 2.28 \\
\hline & & $(\mathrm{U}-\mathrm{N})_{\mathrm{av} .}$ & 2.291 & 0.60 & 0.89 & 0.99 & 1.11 & 0.96 & 0.76 \\
\hline
\end{tabular}

${ }^{a}$ Gophinatan-Jug bond order analysis. ${ }^{b}$ Nalewajski-Mrozek bond order analysis based on different partitionings. 
增加 0.09 , 而其他轨道则减少 $0.06 \sim 0.16$. GGA 方法表 明电子更容易定域于 $5 \mathrm{f}$ 轨道, 且分子环境(溶液和气态) 对原子布居几乎没有影响. 杂化泛函方法得到不同的结 果, 如二价铀配合物的计算表明杂化泛函方法相对 GGA 方法低估了电子在 $5 \mathrm{f}$ 轨道中的分布, 其计算数值 降低了 $0.17 \sim 0.20$, 而高估了电子在 $6 \mathrm{~s}$ 和 $7 \mathrm{~s}$ 轨道中的占 据, 相对增加共计 $0.29 \sim 0.31$ 个电子. 这一结果与密度 泛函方法的本质密切相关 ${ }^{[16,35 ~ 37]}$.

表 $3 \mathrm{ADF}$ 程序计算的配合物基态中铀的电子自旋密度 $\left(S_{\mathrm{U}}\right)$ 、电荷 $\left(Q_{\mathrm{U}}\right)$ 和原子布居

Table 3 Electron-spin density $\left(S_{\mathrm{U}}\right)$, charge $\left(Q_{\mathrm{U}}\right)$ and atomic populations of the uranium atom in the diuranium complexes in their ground states calculated by the ADF code

\begin{tabular}{ccccc}
\hline Complex & Approach & $S_{\mathrm{U}}$ & $Q_{\mathrm{U}}$ & Atomic population \\
\hline$\left[\left(\mathrm{U}^{\mathrm{II}}\right)_{2}(\mathrm{~L})\right]$ & PBE/sol & 0.955 & 1.070 & $5 \mathrm{f}^{3.15} 6 \mathrm{~s}^{1.90} 6 \mathrm{p}^{6.00} 6 \mathrm{~d}^{1.82} 7 \mathrm{p}^{0.06}$ \\
& PBE/gas & 0.951 & 1.092 & $5 \mathrm{f}^{3.15} 6 \mathrm{~s}^{1.90} 6 \mathrm{p}^{6.00} 6 \mathrm{~d}^{1.82} 7 \mathrm{p}^{0.04}$ \\
& B3LYP/sol & 0.931 & 1.044 & $5 \mathrm{f}^{2.98} 6 \mathrm{~s}^{2.00} 6 \mathrm{p}^{5.95} 6 \mathrm{~d}^{1.75} 7 \mathrm{~s}^{0.27}$ \\
& $\mathrm{mPW}$ 1PW/sol & 0.936 & 1.067 & $5 \mathrm{f}^{2.95} 6 \mathrm{~s}^{2.00} 6 \mathrm{p}^{5.95} 6 \mathrm{~d}^{1.78} 7 \mathrm{~s}^{0.25}$ \\
{$\left[\left(\mathrm{U}^{\mathrm{III}}\right)_{2}(\mathrm{~L})\right]^{2+}$} & PBE/sol & 1.111 & 1.448 & $5 \mathrm{f}^{3.04} 6 \mathrm{~s}^{1.88} 6 \mathrm{p}^{5.84} 6 \mathrm{~d}^{1.80}$ \\
{$\left[\left(\mathrm{U}^{\mathrm{IV}}\right)_{2}(\mathrm{~L})\right]^{4+}$} & PBE/sol & 2.132 & 1.710 & $5 \mathrm{f}^{2.98} 6 \mathrm{~s}^{1.97} 6 \mathrm{p}^{5.70} 6 \mathrm{~d}^{1.64}$ \\
\hline
\end{tabular}

\section{3 分子轨道和金属多重键}

使用溶剂化自洽反应场模型模拟分子环境, 计算三 种铀氧化态配合物的基态电子性质, 同时与三价铀配合 物的五重态的进行比较. 图 3 绘制 $\alpha$ 自旋轨道能级, 并 标明轨道性质. 它们的定量轨道成分和轨道图见支持信 息表 $\mathrm{S} 2 \sim \mathrm{S} 4$ 和图 $\mathrm{S} 1 \sim \mathrm{S} 3$.

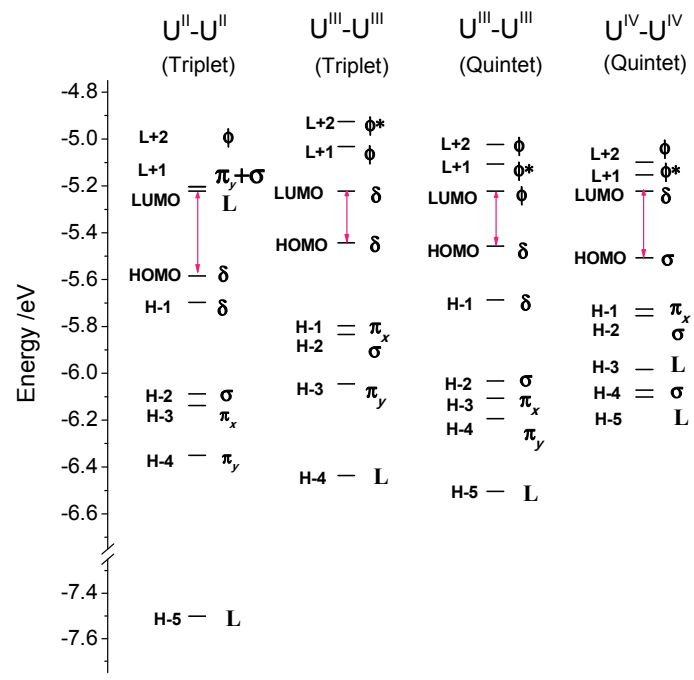

图 $3 \mathrm{PBE} / \mathrm{Scalar}-\mathrm{ZORA} / \mathrm{sol}(\mathrm{THF})$ 方法计算 $\left[\left(\mathrm{U}^{m}\right)_{2}(\mathrm{~L})\right]^{n+}(m=\mathrm{II} \sim \mathrm{IV} ; n$ $=0 \sim 4$ )基态的 $\alpha$ 自旋轨道能级, 并与三价配合物的五重态进行比较. 为方便, 二价配合物的所有轨道整体下移 $2.12 \mathrm{eV}$, 三价配合物的三重 态整体上移 $0.05 \mathrm{eV}$. 而四价配合物的向上平移 $2.01 \mathrm{eV}$

Figure 3 Energetic levels and character of frontier molecular orbitals $(\alpha$-spin $)$ of $\left[\left(\mathrm{U}^{m}\right)_{2}(\mathrm{~L})\right]^{n+}(m=\mathrm{II} \sim \mathrm{IV} ; n=0 \sim 4)$ in ground states calculated by PBE/Scalar-ZORA/sol(THF), compared with those of the second lowest-state $\left[\left(\mathrm{U}^{\mathrm{III}}\right)_{2}(\mathrm{~L})\right]^{2+}$ (quintet). For convenient comparison, overall orbitals of the divalent complex (first column) were down-shifted by 2.12 $\mathrm{eV}$, those of the triplet state of the trivalent complex (second column) up-shifted by $0.05 \mathrm{eV}$, while those of the tetravalent complex (fourth column) up-shifted by $2.01 \mathrm{eV}$
图 3 显示，这些配合物拥有金属性质的前线分子轨 道(包括高占据轨道和低非占据轨道), 配体贡献为主的 占据轨道能量相对较低. 有趣的是, 最高配体占据轨道 与其相邻的低金属占据轨道之间能量差逐渐缩小.二价 铀配合物的 HOMO-LUMO 轨道能级差为 $1.15 \mathrm{eV}$, 三 价铀配合物的能量差减小至 $0.39 \mathrm{eV}$ (三重态)和 0.31 $\mathrm{eV}$ (五重态), 然而四价铀配合物的最高能配体轨道能量 急剧升高, 并超过 $\sigma(\mathrm{U}-\mathrm{U})$ 金属轨道 $0.09 \mathrm{eV}$, 即其 $\mathrm{HOMO}-3(\mathrm{H}-3)$ 配体轨道插入到两个金属 $\sigma$ 成键轨道 $\mathrm{H}-4$ 和 $\mathrm{H}-2$ 之间. 在以前系列配合物 $\mathrm{An}^{m} \mathrm{Cp}_{n}(\mathrm{Cp}=$ $\left.\mathrm{C}_{5} \mathrm{H}_{5} ; \mathrm{An}=\mathrm{Th} \sim \mathrm{Cm} ; m=\mathrm{III}, n=3 ; m=\mathrm{IV}, n=4\right)$ 研究 中 $^{[38,39]}$, 发现随着钣系金属原子序数增加, 最高能配体 占据轨道能量逐渐接近、并在超钚元素时高过相邻最低 能金属轨道能级; 得出金属和配体轨道能级匹配可以在 一定程度上增大配体与金属相互作用的结论，从而为次 钣元素(如 $\mathrm{Am}$ 和 $\mathrm{Cm}$ 等)分离化学提供理论指导 ${ }^{[40]}$. 本 文研究发现, 金属铀氧化态变化同样能够调控配体和金 属轨道能级匹配程度和影响其相互作用. 值得指出的 是, Priroad/ADF 对二价、三价和四价铀配合物基态的 $\mathrm{U}-\mathrm{N}$ 的 Mayer 键级计算分别为 $0.51 / 0.39 、 0.63 / 0.46$ 和 $0.76 / 0.60$, 即随着铀氧化态增加而 $\mathrm{U}-\mathrm{N}$ 键级增大, 这 一结果直接证明配体与金属的成键作用增强. $\mathrm{U}-\mathrm{N}$ 的 G-J 和 N-M(i)键级给出同样的规律(表 2).

二价铀配合物具有三重态基态，它的 HOMO 和 $\mathrm{H}-1$ ( $\alpha$ 自旋)轨道为 $\delta(\mathrm{U}-\mathrm{U})$ 成键性质, 如图 4 所示, 分 别具有一个成单电子, 且自旋平行. 能量较低的 $\sigma$ 和 $\pi$ 轨道均为双占据轨道. 因此, 二价铀配合物具有 $\pi_{y}{ }^{2} \pi_{x}{ }^{2} \sigma^{2} \delta^{1} \delta^{1}$ 电子组态. 表 4 和图 5 给出了 $\alpha$ 自旋前线轨 道成分分析. 很明显, 该配合物有五个高占据金属性质 轨道, $\mathrm{HOMO} \sim \mathrm{H}-4$. 它们主要为 $\mathrm{U}(\mathrm{f})$ 轨道成分, 并有 部分 $U(d)$ 轨道特征, 其中 $\mathrm{H}-4$ 中有 $29 \% \mathrm{U}(\mathrm{d})$ 贡献; $\mathrm{H}-$ 2 中还有 $13 \% \mathrm{U}(\mathrm{s})$ 和 $7 \%$ 的 $U(\mathrm{p})$ 参与. $\mathrm{H}-5$ 主要为配体 参与的轨道 $(77 \%)$.
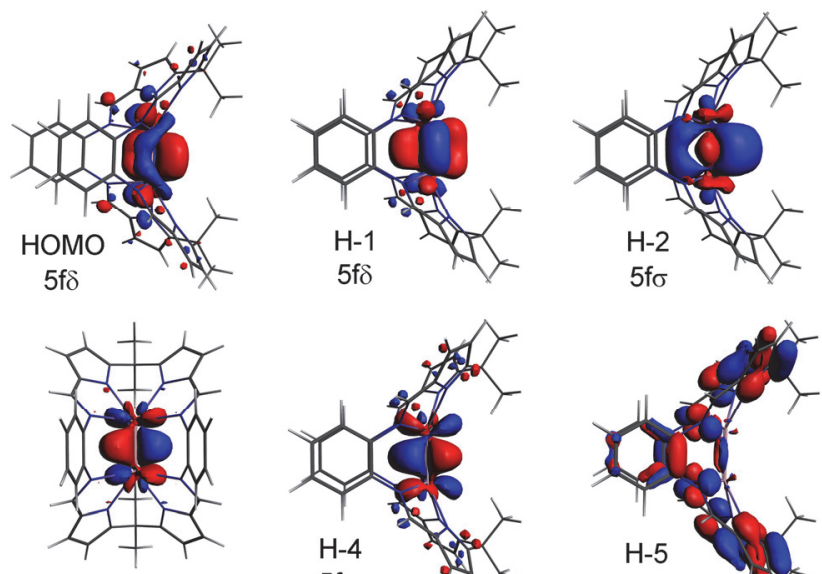

H-3 $5 f \pi_{x}$
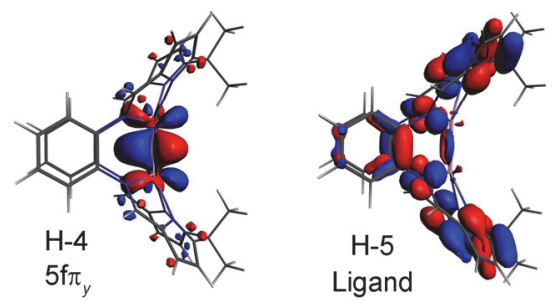

图 4 配合物 $\left[\left(\mathrm{U}^{\mathrm{II}}\right)_{2}(\mathrm{~L})\right]$ 基态的 $\mathrm{U}(\mathrm{f})$ 和配体性质轨道 $(\alpha$ 自旋)

Figure 4 Characteristic $U(f)$ and ligand-based $\alpha$-spin orbitals of the ground (triplet) state of $\left[\left(\mathrm{U}^{\mathrm{II}}\right)_{2}(\mathrm{~L})\right]$ 
表 4 配合物 $\left[\left(\mathrm{U}^{\mathrm{II}}\right)_{2}(\mathrm{~L})\right]$ 基态的 $\alpha$ 自旋轨道成分

Table 4 Contributions (\%) to the $\alpha$-spin orbitals of the ground (triplet) state of $\left[\left(\mathrm{U}^{\mathrm{II}}\right)_{2}(\mathrm{~L})\right]^{a}$

\begin{tabular}{|c|c|c|c|c|c|c|}
\hline \multirow{2}{*}{ Orbital } & \multirow{2}{*}{$\begin{array}{c}\text { Energy/ } \\
\mathrm{eV}\end{array}$} & \multicolumn{4}{|c|}{$2 \mathrm{U}^{b}$} & \multirow{2}{*}{ Ligand } \\
\hline & & $\mathrm{s}$ & $\mathrm{p}$ & d & $\mathrm{f}$ & \\
\hline $\mathrm{L}+2$ & -2.719 & & & & 90.5 & \\
\hline $\mathrm{L}+1$ & -2.720 & 8.7 & & 8.9 & 42.3 & 12.3 \\
\hline LUMO & -2.737 & & & 4.1 & 6.8 & 56.6 \\
\hline HOMO & -3.100 & & & 2.2 & 79.7 & \\
\hline $\mathrm{H}-1$ & -3.214 & & & 12.9 & 75.1 & \\
\hline $\mathrm{H}-2$ & -3.604 & 12.9 & 7.0 & 7.2 & 64.2 & \\
\hline $\mathrm{H}-3$ & -3.654 & & & 18.1 & 67.2 & \\
\hline $\mathrm{H}-4$ & -3.867 & & & 29.1 & 48.8 & \\
\hline $\mathrm{H}-5$ & -5.017 & & & 2.3 & & 77.1 \\
\hline
\end{tabular}

${ }^{a}$ The sum of contributions for each orbital is less than $100 \%$, for the output of SFO contributions smaller than $1 \%$ in the calculations is suppressed. ${ }^{b}$ Total values of two uranium atoms are given here, for each uranium has approximately identical components.

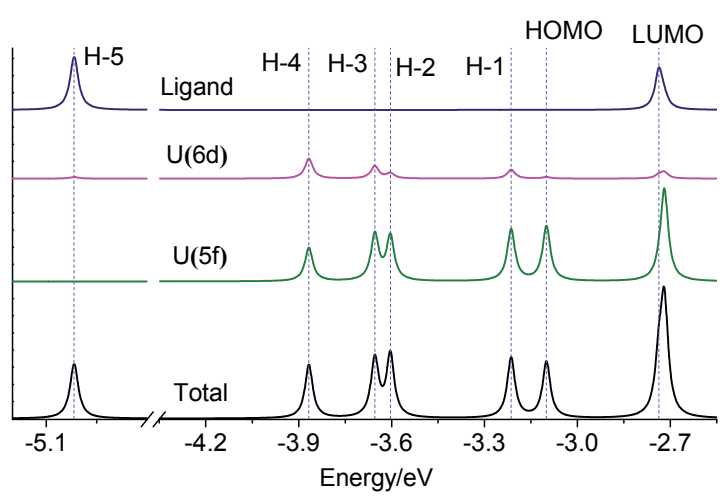

Figure 5 Density of states (DOS) of $\alpha$-spin orbitals of the triplet state of $\left[\left(\mathrm{U}^{\mathrm{II}}\right)_{2}(\mathrm{~L})\right]$

图 5 三重态 $\left[\left(\mathrm{U}^{\mathrm{II}}\right)_{2}(\mathrm{~L})\right] \alpha$ 自旋轨道的态密度图

三价铀配合物基态高占据轨道 $(\mathrm{HOMO} \sim \mathrm{H}-3)$ 分 别为金属铀-铀的 $\delta 、 \sigma$ 和 $\pi$ 成键轨道, 具有 $\pi_{y}{ }^{2} \sigma^{2} \pi_{x}{ }^{1} \delta^{1}$ 电子组态. 这些轨道仍以 $U(5 f)$ 成分为主, $U(6 d)$ 贡献相 对二价铀配合物都有所减小, 其中 $\mathrm{H}-3$ 轨道的 $\mathrm{U}(6 \mathrm{~d})$ 成分最大, 不超过 $13 \%$. 计算表明四价铀配合物拥有 $\sigma^{1} \sigma^{1} \pi_{x}{ }^{1} \sigma^{1}$ 电子组态. 与上面两个配合物相比, 四价配合 物的轨道成分发生较大变化. 如前所述, 配体轨道能量 升高并超过金属轨道. 这导致其占据轨道主要由 $U$ 参与 为主, 而配体部分参与成键. 例如, $\mathrm{H}-1$ 和 $\mathrm{H}-2$ 中分 别有 $23 \%$ 和 $21 \%$ 的配体贡献. 另外, 金属成键轨道 (HOMO、 $\mathrm{H}-1 、 \mathrm{H}-2$ 和 $\mathrm{H}-4$ ) 完全是 $\mathrm{U}(5 \mathrm{f})$ 性质，而 $\mathrm{U}(6 \mathrm{~d})$ 仅有不超过 $3 \%$ 的贡献.

使用 $\mathrm{ADF}$ 和 Multiwfn 程序计算 QTAIM 性质参数, 电子密度 $\rho(r)$ 、拉普拉斯密度 $\nabla^{2} \rho(r)$ 和能量密度 $H(r)$. 对 于二价铀配合物, 除九重态外, 所有的 $\rho(r)$ 均大于 0.1 , 而 $\nabla^{2} \rho(r)$ 大于 0.34 , 其 $H(r)$ 为负值, 说明二价 $\mathrm{U}$ 配合物 的 $\mathrm{U}-\mathrm{U}$ 键具有部分共价特征. 其中, 三重基态的计算 值分别为 $0.19 、 0.52$ 和 -0.11 . 计算表明, QTAIM 参数 数值(绝对值) 随铀氧化态的增加而逐渐减小, 说明铀-
铀金属键强度逐渐减小. 结合以上 $\mathrm{U}-\mathrm{U}$ 键长、键级、 伸缩振动频率和分子轨道分析, 二价、三价和四价铀配 合物分别具有 $U-U$ 弱四重键、三重键和双重键性质.

表 5 双金属铀配合物中 $\mathrm{U}-\mathrm{U}$ 键临界点电子密度参数: $\rho(r) 、 \nabla^{2} \rho(r)$ 、 $H(r)$ (单位: $a u)$

Table 5 Electron density $\left[\rho(r)\right.$, au], its Laplacian $\left[\nabla^{2} \rho(r), a u\right]$ and energy density $[H(r), a u]$ at $\mathrm{U}-\mathrm{U}$ bond critical point in diuranium complexes, where all electronic states were calculated for the divalent uranium complex, and only the ground state for other complexes

\begin{tabular}{ccccc}
\hline Complex & ES & $\rho(r)$ & $\nabla^{2} \rho(r)$ & $H(r)$ \\
\hline$\left[\left(\mathrm{U}^{\mathrm{II}}\right)_{2}(\mathrm{~L})\right]$ & nonet & 0.088 & 0.266 & -0.030 \\
& septet & 0.122 & 0.343 & -0.049 \\
& quintet & 0.169 & 0.344 & -0.067 \\
& triplet & 0.189 & 0.517 & -0.109 \\
& singlet & 0.191 & 0.552 & -0.112 \\
{$\left[\left(\mathrm{U}^{\mathrm{III}}\right)_{2}(\mathrm{~L})\right]^{2+}$} & triplet & 0.125 & 0.421 & $-{ }^{a}$ \\
{$\left[\left(\mathrm{U}^{\mathrm{IV}}\right)_{2}(\mathrm{~L})\right]^{4+}$} & quintet & 0.082 & 0.251 & -0.026 \\
\hline
\end{tabular}

${ }^{a}$ Don't obtain the value.

\section{4 结论}

使用相对论广义梯度和杂化密度泛函理论方法探 索二价双金属铀配合物的各种电子自旋态结构、电子自 旋密度、基态电子结构和金属多重键性质，并与其三价 和四价铀同类物进行比较.

优化得到二价铀配合物有五种电子自旋态, 其三重 态为基态，不同泛函的计算得到相同结论. 三价和四价 铀化合物的基态分别为三重和五重态.二价、三价和四 价铀化合物的基态 $\mathrm{U}-\mathrm{U}$ 键长分别为 $2.33 、 2.47$ 和 2.72 $\AA$, Mayer 键级为 3.89、3.18 和 2.28, 伸缩振动频率为 $259 、 214$ 和 $140 \mathrm{~cm}^{-1}$; 并分别具有 $\pi_{y}^{2} \pi_{x}^{2} \sigma^{2} \delta^{1} \delta^{1} 、 \pi_{y}{ }^{2} \sigma^{2} \pi_{x}{ }^{1} \delta^{1}$ 和 $\sigma^{1} \sigma^{1} \pi_{x}^{1} \sigma^{1}$ 电子组态. 结合 QTAIM 拓扑分析, 指认二 价、三价和四价双铀配合物分别具有弱 $U-U$ 四重键、 三重键和双重键本质.

分子轨道分析表明二价铀配合物的 $\mathrm{U}-\mathrm{U}$ 成键的高 占据轨道, 主要由 $U(5 f)$ 贡献且混有部分 $U(6 \mathrm{~d})$ 成分; 其 最高能配体占据轨道与上面相邻的最低能金属轨道能 量相差 $1.15 \mathrm{eV}$. 三价铀配合物的高占据轨道成分仍以 $\mathrm{U}(5 \mathrm{f})$ 为主, $\mathrm{U}(6 \mathrm{~d})$ 贡献相对减小; 金属轨道与最高占据 配体轨道能量差仅为 $0.39 \mathrm{eV}$. 相比之下, 四价铀配合物 的电子结构发生较大变化. 首先配体轨道能量急剧升 高, 并超过金属轨道 $0.09 \mathrm{eV}$; 其次, 配体对轨道成分贡 献相对二价和三价配合物明显增大; 最后, 金属成键轨 道完全为 $\mathrm{U}(5 \mathrm{f})$ 性质, $\mathrm{U}(6 \mathrm{~d})$ 贡献可以忽略.

目前研究发现随着铜系金属原子序数的增加, 最高 配体占据轨道能量逐渐接近、并在超钚元素配合物时超 越相邻最低金属轨道能级的现象, 这归结为金属和配体 轨道能级匹配是它们相互作用增强的主因 ${ }^{[38,39]}$. 本文研 究发现，通过变化金属铀的氧化态，同样可以实现配体 和金属轨道能级匹配程度的有效调控，并增加金属和配 体的相互作用. 


\section{References}

[1] Hashke, J. M.; Stakebake, J. L. In The Chemistry of the Actinide and Transactinide Elements, Eds.: Morss, L. R.; Edelstein, N. M.; Fuger, J., Springer, Netherlands, 2006, p. 3199.

[2] Liddle, S. T. Angew. Chem. Int. Ed. 2015, 54, 8604.

[3] MacDonald, M. R.; Fieser, M. E.; Bates, J. E.; Ziller, J. W.; Furche, F.; Evans, W. J. J. Am. Chem. Soc. 2013, 135, 13310.

[4] La Pierre, H. S.; Scheurer, A.; Heinemann, F. W.; Hieringer, W.; Meyer, K. Angew. Chem. Int. Ed. 2014, 53, 7158.

[5] Meyer, G. Angew. Chem. Int. Ed. 2014, 53, 3550.

[6] Sessler, J. L.; Cho, W. S.; Dudek, S. P.; Hicks, L.; Lynch, V. M.; Huggins, M. T. J. Porphyr. Phthalocyanines 2003, 7, 97.

[7] Givaja, G.; Blake, A. J.; Wilson, C.; Schroder, M.; Love, J. B. Chem. Commun. 2003, 2508.

[8] Arnold, P. L.; Patel, D.; Wilson, C.; Love, J. B. Nature 2008, 451, 315.

[9] Arnold, P. L.; Potter, N. A.; Magnani, N.; Apostolidis, C.; Griveau, J.-C.; Colineau, E.; Morgenstern, A.; Caciuffo, R.; Love, J. B. Inorg. Chem. 2010, 49, 5341

[10] Arnold, P. L.; Potter, N. A.; Carmichael, C. D.; Slawin, A. M. Z.; Roussel, P.; Love, J. B. Chem. Commun. 2010, 46, 1833.

[11] Arnold, P. L.; Hollis, E.; White, F. J.; Magnani, N.; Caciuffo, R.; Love, J. B. Angew. Chem. Int. Ed. 2011, 50, 887.

[12] Arnold, P. L.; Jones, G. M.; Odoh, S. O.; Schreckenbach, G.; Magnani, N.; Love, J. B. Nat. Chem. 2012, 4, 221.

[13] Zegke, M.; Nichol, G. S.; Arnold, P. L.; Love, J. B. Chem. Commun. 2015, 51, 5876.

[14] Su, D.-M.; Zheng, X.-J.; Schreckenbach, G.; Pan, Q.-J. Organometallics 2015, 34, 5225.

[15] Laikov, D. N.; Ustynyuk, Y. A. Russ. Chem. Bull. 2005, 54, 820.

[16] Perdew, J. P.; Burke, K.; Ernzerhof, M. Phys. Rev. Lett. 1996, 77, 3865.

[17] Baerends, E. J.; Ziegler, T.; Autschbach, J.; Bashford, D.; Bérces, A.; Bickelhaupt, F. M.; Bo, C.; Boerrigter, P. M.; Cavallo, L.; Chong, D. P.; Deng, L.; Dickson, R. M.; Ellis, D. E.; van Faassen, M.; Fan, L.; Fischer, T. H.; Fonseca Guerra, C.; Franchini, M.; Ghysels, A.; Giammona, A.; van Gisbergen, S. J. A.; Götz, A. W.; Groeneveld, J. A.; Gritsenko, O. V.; Grüning, M.; Gusarov, S.; Harris, F. E.; van den Hoek, P.; Jacob, C. R.; Jacobsen, H.; Jensen, L.; Kaminski, J. W.; van Kesse, G.; Kootstra, F.; Kovalenko, A.; Krykunov, M. V.; van Lenthe, E.; McCormack, D. A.; Michalak, A.; Mitoraj, M.; Morton, S. M.; Neugebauer, J.; Nicu, V. P.; Noodleman, L.; Osinga, V. P.; Patchkovskii, S.; Pavanello, M.; Philipsen, P. H. T.; Post, D.; Pye, C. C.; Ravenek, W.; Rodríguez, J. I.; Ros, P.; Schipper, P. R. T.; van Schoot, H.; Schreckenbach, G.; Seldenthuis, J. S.; Seth, M.; Snijders, J. G.; Solà, M.; Swart, M.; Swerhone, D.; te Velde, G.; Vernooijs, P.; Versluis, L.; Visscher, L.; Visser, O.; Wang, F.; Wesolowski, T. A.; van Wezenbeek, E. M.; Wiesenekker, G.; Wolff, S. K.; Woo, T. K.; Yakovlev, A. L.; ADF2014.06 ed.; SCM, Theoretical Chemistry, Vrije Universiteit: Amsterdam, The Netherlands, 2014.

[18] Klamt, A.; Jonas, V.; Burger, T.; Lohrenz, J. C. W. J. Phys. Chem. A 1998, 102, 5074 .
[19] Bao, Z.; Zhao, H.-B.; Qu, N.; Schreckenbach, G.; Pan, Q.-J. Dalton Trans. 2016, 45, 15970 .

[20] Zhao, S.; Zhong, Y.; Guo, Y.; Zhang, H.; Pan, Q. Acta Chim. Sinica 2016, 74, 683. (赵思魏, 钟宇曦, 郭元茹, 张红星, 潘清江, 化学 学报, 2016, 74, 683.)

[21] van Lenthe, E.; Baerends, E. J.; Snijders, J. G. J. Chem. Phys. 1993, 99, 4597.

[22] Frisch, M. J.; Trucks, G. W.; Schlegel, H. B.; Scuseria, G. E.; Robb, M. A.; Cheeseman, J. R.; Scalmani, G.; Barone, V.; Mennucci, B.; Petersson, G. A.; Nakatsuji, H.; Caricato, M.; Li, X.; Hratchian, H. P.; Izmaylov, A. F.; Bloino, J.; Zheng, G.; Sonnenberg, J. L.; Hada, M.; Ehara, M.; Toyota, K.; Fukuda, R.; Hasegawa, J.; Ishida, M.; Nakajima, T.; Honda, Y.; Kitao, O.; Nakai, H.; Vreven, T.; Montgomery, J. A., Jr.; Peralta, J. E.; Ogliaro, F.; Bearpark, M.; Heyd, J. J.; Brothers, E.; Kudin, K. N.; Staroverov, V. N.; Kobayashi, R.; Normand, J.; Raghavachari, K.; Rendell, A.; Burant, J. C.; Iyengar, S. S.; Tomasi, J.; Cossi, M.; Rega, N.; Millam, J. M.; Klene, M.; Knox, J. E.; Cross, J. B.; Bakken, V.; Adamo, C.; Jaramillo, J.; Gomperts, R.; Stratmann, R. E.; Yazyev, O.; Austin, A J.; Cammi, R. P., C.; Ochterski, J. W.; Martin, R. L.; Morokuma, K.; Zakrzewski, V. G.; Voth, G. A.; Salvador, P.; Dannenberg, J. J.; Dapprich, S.; Daniels, A. D.; Farkas, O.; Foresman, J. B.; Ortiz, J. V.; Cioslowski, J.; Fox, D. J.; Gaussian 09, Revision D.01 ed. Gaussian, Inc., Wallingford CT, 2009.

[23] Cao, X.; Dolg, M.; Stoll, H. J. Chem. Phys. 2003, 118, 487.

[24] Lu, T.; Chen, F. J. Comput. Chem. 2012, 33, 580.

[25] Patel, D.; Liddle, S. T. Rev. Inorg. Chem. 2012, 32, 1.

[26] Patel, D.; King, D. M.; Gardner, B. M.; McMaster, J.; Lewis, W.; Blake, A. J.; Liddle, S. T. Chem. Commun. 2011, 47, 295.

[27] Gardner, B. M.; Patel, D.; Cornish, A. D.; McMaster, J.; Lewis, W.; Blake, A. J.; Liddle, S. T. Chem. Eur. J. 2011, 17, 11266.

[28] Liddle, S. T.; McMaster, J.; Mills, D. P.; Blake, A. J.; Jones, C.; Woodul, W. D. Angew. Chem. Int. Ed. 2009, 48, 1077.

[29] Hlina, J. A.; Pankhurst, J. R.; Kaltsoyannis, N.; Arnold, P. L. J. Am. Chem. Soc. 2016, 138, 3333.

[30] Cavigliasso, G.; Kaltsoyannis, N. Inorg. Chem. 2006, 45, 6828

[31] Cavigliasso, G.; Kaltsoyannis, N. Dalton Trans. 2006, 5476.

[32] Roos, B. O.; Gagliardi, L. Inorg. Chem. 2006, 45, 803.

[33] Zhou, J.; Sonnenberg, J. L.; Schlegel, H. B. Inorg. Chem. 2010, 49, 6545 .

[34] Long, B.; Bao, J. L.; Truhlar, D. G. J. Am. Chem. Soc. 2016, 138, 14409.

[35] Xiong, Z.; Chen, Q.; Zheng, X.; Wei, X. Acta Chim. Sinica 2005, 63, 572. (熊忠华, 陈琦, 郑秀梅, 魏锡文, 化学学报, 2005, 63, 572.)

[36] Zhang, Y.; Ma, X.; Zhang, X.; Lei, M. Acta Chim. Sinica 2016, 74, 340. (张益伟, 马雪璐, 张欣, 雷鸣, 化学学报, 2016, 74, 340.)

[37] Yang, Y.; Zhang, Q.; Shi, J.; Fu, Y. Acta Chim. Sinica 2016, 74, 422. (杨一诺, 张琪, 石景, 傅尧, 化学学报, 2016, 74, 422.)

[38] Kirker, I.; Kaltsoyannis, N. Dalton Trans. 2011, 40, 124.

[39] Tassell, M. J.; Kaltsoyannis, N. Dalton Trans. 2010, 39, 6719.

[40] Liu, J.-B.; Chen, G. P.; Huang, W.; Clark, D. L.; Schwarz, W. H. E.; Li, J. Dalton Trans. 2017, 46, 2542.

(Cheng, B.) 


\section{Structures and Metal-Metal Multiple \\ Bond of Binuclear Divalent Uranium Complex of Pyrrolic Schiff-base Mac- rocycle: a Relativistic DFT Probe}

Chen, Fangyuan; Qu, Ning; Wu, Qunyan; Zhang, Hongxing; Shi, Weiqun*; Pan, Qingjiang*

Acta Chim. Sinica 2017, 75(X), XXX-XXX
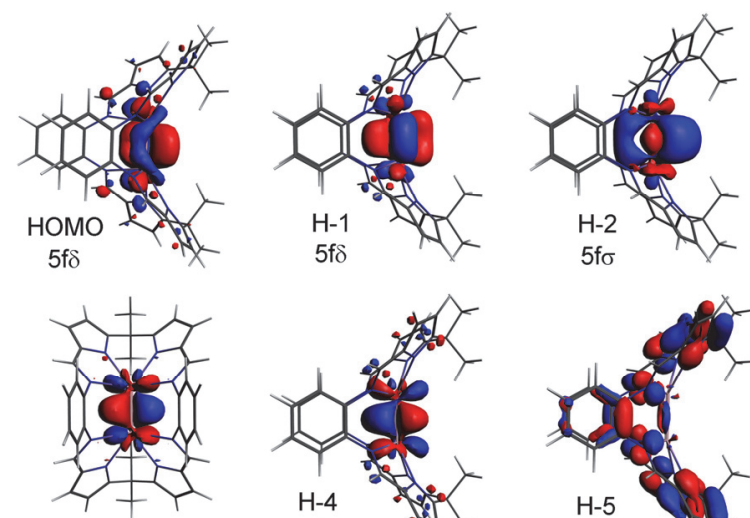

$\mathrm{H}-35 \pi_{x}$
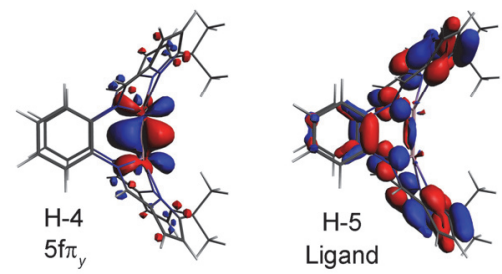

A relativistic DFT probe has been carried out on the divalent diuranium complex of a single macrocyclic ligand. Its triplet state was found as the ground state. Structural parameters, molecular orbitals and QTAIM analysis all indicate diuranium multiple bonding nature. Comparison with tri- and tetravalent diuranium analogues finds that the uranium oxidation state can tune energetic match between the highest-energy occupied ligand orbital and the adjacent low-energy metal-based orbital, as well as correlates with the electron transfer between metal and ligand and the diuranium multiple bond number. 